\title{
All-optical wavelength conversion based on degenerate four-wave mixing in Raman ring laser.
}

\begin{abstract}
We demonstrate an all-optical wavelength converter using a ring cavity that produces conversion efficiency of more than unity within a certain bandwidth. The highly nonlinear fiber is used as the parametric amplifying medium and it is pumped by four independent pump lasers at $1427,1443,1461$, and $1493 \mathrm{~nm}$. The parametric pump is formed internally from the ring cavity through the effect of stimulated Raman scattering. The interaction between signal and parametric pump becomes intensified as they propagate along the fiber from input to the output ports. This un-depleted pump power condition enhances the process of degenerate four-wave mixing in the proposed structure. The maximum conversion efficiency is obtained at $9.5 \mathrm{~dB}$ and the bandwidth of positive conversion efficiency is about $20.6 \mathrm{~nm}$.
\end{abstract}

Keyword: Four-wave mixing; Raman amplification; Fiber laser. 